G. C. Montanari, P. Seri, M. Paajanen, K. Lahti, I. Rytöluoto, M. Ritamäki, A. Blume, W. Dierkes, A. Mahtabani, X. He, "Potentiality of nanofilled thermoplastic insulation for DC cables and capacitors," 2018 IEEE 2nd International Conference on Dielectrics (ICD), Budapest, 2018, pp. 1-4.

The published version is available online at:

https://doi.org/10.1109/ICD.2018.8514726

(C) 2018 IEEE. Personal use of this material is permitted. Permission from IEEE must be obtained for all other uses, in any current or future media, including reprinting/republishing this material for advertising or promotional purposes, creating new collective works, for resale or redistribution to servers or lists, or reuse of any copyrighted component of this work in other works. 


\section{Potentiality of nanofilled thermoplastic insulation for DC cables and capacitors}

\author{
Gian Carlo Montanari \\ $D E I$ \\ University of Bologna \\ Bologna, Italy \\ Center for Electromechanics \\ University of Texas \\ Austin, Texas \\ Paolo Seri \\ DEI \\ University of Bologna \\ Bologna, Italy \\ Mika Paajanen \\ VTT Technical Research Centre of Finland Ltd \\ Tampere, Finland
}

\author{
Kari Lahti \\ Ilkka Rytöluoto \\ Mikael Ritamäki \\ Electrical Energy Engineering \\ Tampere University of Technology \\ Tampere, Finland \\ Anke Blume \\ Wilma Dierkes \\ Amirhossein Mahtabani \\ Xiaozhen He \\ Faculty of Engineering Technology \\ University of Twente \\ Enschede, The Netherlands
}

\begin{abstract}
This paper presents space charge accumulation characteristics of cable grade and capacitor grade nanostructured polypropylene (PP) materials, providing initial results regarding the electrical properties of nanofilled PP materials belonging to the European project GRIDABLE. This project has the aim to develop DC cable extruded insulation and $M V$ and $L V D C$ capacitor films having enhanced performance with respect to presently used materials. The paper shows that nanostructuration may be beneficial, especially at higher temperature, to improve the capacitor-grade material performance. On the other hand, this work validates the importance of surface functionalization of nanofillers, leading to lower space charge accumulation on DC cable-grade materials.
\end{abstract}

Keywords-Nanodielectrics; Polypropylene; HVDC; space charge

\section{INTRODUCTION}

The trend towards more environmentally compatible electrical technologies is growing the investments in DC electrical apparatus and relevant insulation systems. Focus is, recently, on thermoplastic, thus recyclable, materials. Higher operating electric fields and temperatures would be needed to increase space factor in electrical apparatus design and efficiency, besides specific power. This, however, will involve improving short term and long term performance of the insulating materials which can be used for DC applications. A new European project called GRIDABLE (Polymer Nanocomposite Insulation Material Enabling Integration of Renewables and DC Storage Technologies in the AC Energy Grid) was launched recently under the HORIZON 2020 framework funded by the European Community. Various partners, from universities and research institutions to cable and capacitor manufactures are involved, having the aim to develop a new generation of nanostructured polymeric materials suitable for DC cables and capacitors. The aim of GRIDABLE is to develop Polypropylene-based nanocomposites having proper nanoparticle functionalization and dispersion, demonstrating the improvements this novel material can bring to the state of the art of DC cables and PP capacitors insulation. Under high DC electric fields, the currently used XLPE insulation may experience reliability issues related to the formation of space charge, which can happen through slow charge injection and transport, or through fast charge pulses [1], [2]. In addition, $\mathrm{XLPE}$ is not recyclable and its processing can be energy intensive and complex than other (thermoplastic) materials. While the space charge phenomena can be possibly controlled changing the morphology and crystallinity of the polymer, space charge dispersion can be increased by grafting functional groups onto the nanofiller particle surface, influencing charge mobility through the introduction of low-energy trapping sites on the interaction zones between the nanoparticle and the polymer matrix [3]-[5]. Preliminary work, presented in this paper, indicates that such objectives can be reached, upon proper selection of the base PP and surface treatment of the nanofillers. In the following, space charge test results supporting the developments of the project will be discussed.

\section{ROLE OF SPACE CHARGE IN HVDC APPLICATIONS}

The accumulation of space charge in electrical insulation has been recognized to be a major aging factor in DC insulation [3][5]. Even a moderate increase of electrical field inside the insulation can significantly accelerate the local degradation mechanism driven by electrical stress. Accumulation of space charge can indeed distort and enhance the Laplacian electric 
field distribution inside the insulation. This may bring the maximum electric field amplitude to exceed the design field and considerably shorten the life of the insulation system (even for moderate field enhancements). This is easily demonstrated introducing the relationship between life, L, and the electrical stress, E, described by the inverse power life model (IPM) $[6],[7]:$

$$
L(E)=L_{D}\left(\frac{E}{E_{D}}\right)^{-n}
$$

where $\mathrm{n}$ is the voltage endurance coefficient, $E_{D}$ is design field and $L_{D}$ the corresponding life. Considering the large value of $\mathrm{n}$ (between 15 and 20 at operating temperature, in DC, for materials like XLPE or PP) a small variation of the electric field can translate into a large variation of life by orders of magnitude, e.g. an increase of electric field locally along the insulation thickness of e.g. $10 \%$ can shorten life as much as $75 \%$ [8], [10]. Any material which has to be used as DC insulation, therefore, needs to be characterized to be space charge free (or showing very low amount of space charge) at the operating field and temperature, and nanostructuration might help increasing field and/or temperature above which space charge accumulation becomes large enough to affect field profile and/or accelerate local degradation mechanisms [6],[9].

\section{MATERIAL SPECIFICATION AND COMPOUNDING}

The base material chosen for this project is a thermoplastic material, polypropylene (PP), having excellent electrical and thermal properties for energy applications, and good potentiality regarding mechanical properties, besides being completely recyclable. The choice to add nanofillers, such as $\mathrm{SiO} 2$ with and without functionalization, would fit to the objective to improve electrical and thermal properties and reach higher design field and temperature for the same life and reliability of the present DC technology, that is, XLPE (cross-linked polyethylene) for cables and PP for capacitors.

\section{A. Capacitor-grade cast film materials}

The capacitor grade cast films were produced of commercial capacitor grade isotactic polypropylene homopolymer with 4.5 wt-\% hydrophobic $\mathrm{SiO} 2$ nanoparticle addition and a suitable antioxidant package in preventing oxidative polymer degradation during melt processing. In addition, also an otherwise similar reference cast film was produced without the nanoparticles. The dispersion of the nanoparticles was analyzed based on SEM/TEM microscopy and evaluated to be in general at a good level, although a few agglomerates were observed. More detailed manufacturing details are reported in [11]. The thicknesses of the cast films were in the range of $500 \mu \mathrm{m}$.

\section{B. Cable-grade materials}

The cable compounds were mixed with a Kraussmaffei Berstorff ZE 25/49D twin screw extruder and extrusion of on the 400-500 $\mu \mathrm{m}$ thick sample film was done by Brabender single screw extruder in a soft wall clean room. The nanoparticles were kept in an oven for 1.5 hours at $70^{\circ} \mathrm{C}$ and then additionally for $30 \mathrm{~min}$ at $70^{\circ} \mathrm{C}$ in vacuum to remove any potential moisture.

\section{Surface functionalization of nanofillers}

Nanoparticle surface functionalization is used as an effective way to improve the dispersion of the filler and to establish a strong bond between the polymer and the silica and improve the dielectric properties of the insulation system $[12,13]$. In this study, the silica surface was treated following two different techniques, briefly described in this section. The first functionalized silica batch was prepared with an organosilane as the modifying agent. However, the chosen organosilane contains an amine moiety which is likely to induce deep charge traps in the cable insulation system. The process of silica surface treatment was done in a glass reactor containing the silica particles, $20 \mathrm{w} \%$ organosilane relative to the silica amount, and $0,1 \mathrm{w} \%$ water in a toluene medium at $100^{\circ} \mathrm{C}$ for $24 \mathrm{~h}$. Subsequently, in order to dry the extracted modified silica particles, they were placed in a vacuum oven at $80^{\circ} \mathrm{C}$ for $24 \mathrm{~h}$. The second modified silica batch was prepared using a dry processing method at room temperature for 24 hours. A modifying agent was added to to fumed silica in an alkaline environment in a sealed glass container. The modifying agent was chosen as it is expected to hydrophobize the silica surface and to achieve higher interactions between the filler particles and the PP matrix. The extraction and drying procedures were carried out the same way as mentioned above.

\section{SPACE CHARGE MEASUREMENTS}

Space charge measurements were carried out for both cable and capacitor grade samples by the Pulsed Electro-Acoustic (PEA) method, utilizing $10 \mathrm{~ns}$ pulses with an intensity of $300 \mathrm{~V}$. The acoustic signal is collected by a $9 \mu \mathrm{m}$ thick PVDF piezoelectric and amplified by an amplifier cascade. The thin film system amplifier has a $-3 \mathrm{~dB}$ bandwidth of $250 \mathrm{MHz}$. These features resulted in a spatial resolution of few tens of micrometers. Calibration of the system is made after $10 \mathrm{~s}$ from polarization of the sample, through the following equations:

$$
E(z)=\frac{\int_{0}^{z} \rho(z) d z}{\varepsilon} ; \rho=\varepsilon E_{D C}
$$

where $E(z)$ and $\rho(z)$ are the electric field and the charge density in the thickness of the specimen, $\varepsilon$ is the permittivity of the specimens tested and $E_{D C}$ and $\rho$ are the theoretical electric field and surface charge density when no space charge is accumulated in the sample. Stored charge density at a specific field and depolarization time, $\mathrm{q}(\mathrm{E}, \mathrm{t})$, derived from the space charge profile measurements, as follows:

$$
q(E, t)=\frac{1}{L} \int_{0}^{L}\left|q_{p}(E, x, t) d x\right|
$$

where 0 and $\mathrm{L}$ denote the electrodes positions and $q_{p}(E, x, t)$ is the space charge profile for a given poling field $\mathrm{E}$. Space charge measurements were conducted on cast capacitor grade films and extruded cable grade samples after a conditioning period at $60^{\circ} \mathrm{C}$ under vacuum for 72 hours. Polarization time (when DC voltage is applied) was $10400 \mathrm{~s}$, followed by 3600 seconds of depolarization (when DC voltage is absent). A mechanical switch was used to short the sample in the beginning of the depolarization. Tests were done at $60^{\circ} \mathrm{C}$, in a controlled environment, with fields of $50 \mathrm{kV} / \mathrm{mm}$, to obtain 
indications about performances of those materials under fields exceeding the threshold conditions for space charge accumulation. The chosen stress is well within the operating conditions of HVDC cable materials, while it is much lower than the design for metallized-film capacitors. However, a previous study [15] showed how results of PEA measurements on thick cast films can be in agreement with results obtained with design fields on thin films samples of the same nature.

\section{EXPERIMENTAL RESULTS AND DISCUSSION}

\section{A. Capacitor grade materials}

Figures 1A and 1B show the charge accumulation patterns for capacitor-grade cast film materials, without and with nanofiller. The effect given by the introduction of a $4.5 \%$-wt of nanofiller is highlighted in Figures $1 \mathrm{~A}$ and 1B. During polarization at $60^{\circ} \mathrm{C}$ the PEA system measures a rising trend of charge accumulation for the non-filled material (Figure 1C), while this is less distinct in the filled material (Figure 1D). This is an interesting difference, since it is a clear suggestion that while the filled material has a lower tendency to increase the amount of stored charge over time. Indeed, as can be seen in Figs. $1 \mathrm{E}$ and $1 \mathrm{~F}$, nanofilled materials show higher mobilities (lower trap depth distributions) than the reference (this is shown and modelled in [14]). This is an important characteristic for HVDC materials, since higher charge mobility or lower trap depth distribution contributes to a quicker stored charge removal, therefore a lower field enhancement in the case of polarity inversion and quicker restoration of the material natural charge neutrality.

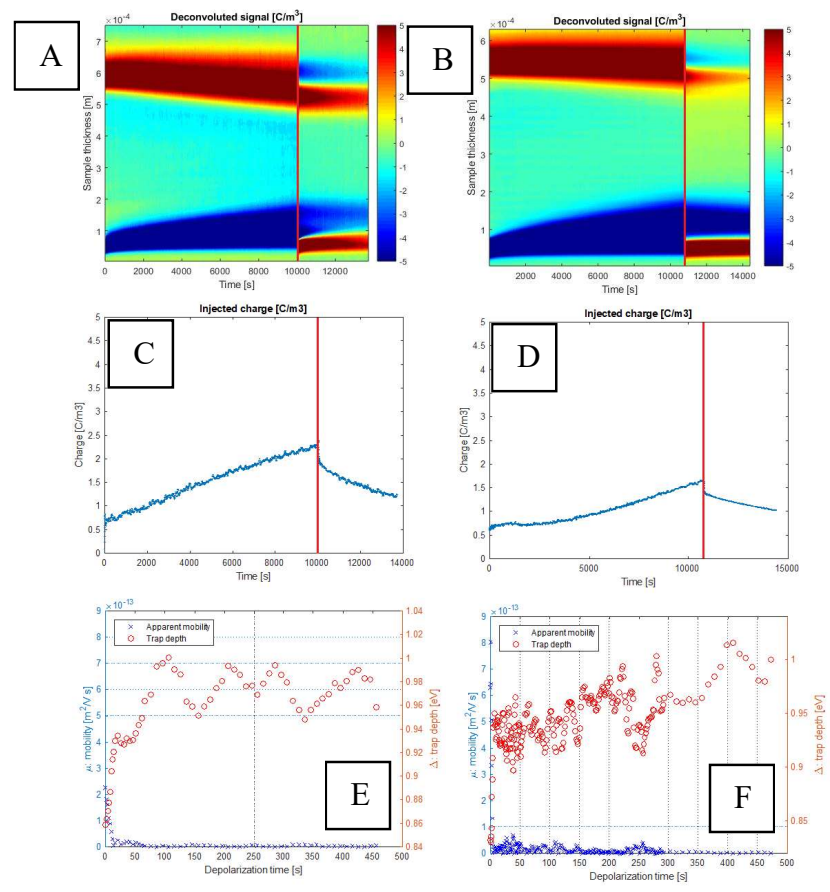

Fig. 1. - Charge patterns, accumulated charge plots and apparent mobility plots for capacitor grade materials (A,C,E: non filled; B,D,F: filled). Depolarization periods starts in correspondence with the red line.
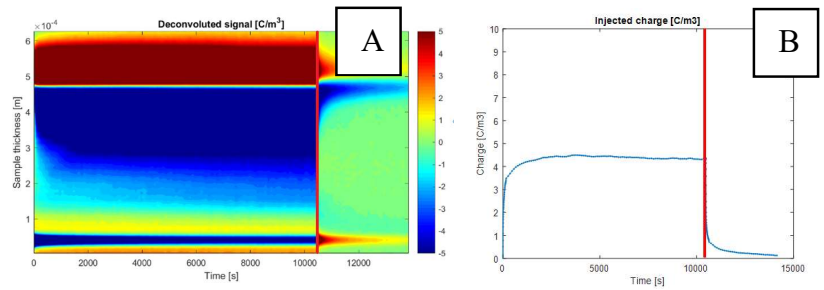

Fig. 2. A: XLPE charge accumulation pattern showing heterocharge accumulation when tested at $50 \mathrm{kV} / \mathrm{mm}, 60^{\circ} \mathrm{C}$. B: XLPE charge accumulation plot under the same conditions. Depolarization periods starts in correspondence with the red line.
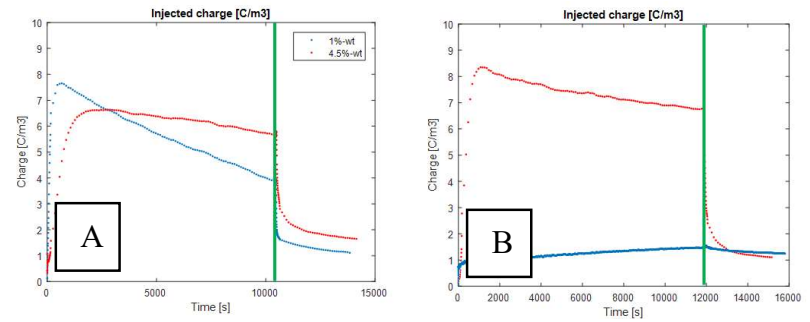

Fig. 3. Charge accumulation plots for two polymeric matrix compositions (A and B) with different filling percentages under $50 \mathrm{kV} / \mathrm{mm}$ poling field at $60^{\circ} \mathrm{C}$. Depolarization periods starts in correspondence with the green line.

\section{B. Cable grade materials}

\section{1) Reference XLPE performance}

Since materials being developed by the GRIDABLE project aim at the technological improvement of the state of the art, that is XLPE, this paragraph briefly describes which is the benchmark provided by this widely used material. As it can be seen from Figure 2, XLPE exhibits a significant heterocharge accumulation at $50 \mathrm{kV} / \mathrm{mm}$, which is, however, two to three times larger than the normally-used design field. After $10400 \mathrm{~s}$ of polarization, an accumulation of $3.2 \mathrm{C} / \mathrm{m}^{3}$ is measured. Calculations on the charge depolarization trends set an apparent mobility of $5 \cdot 10-13 \mathrm{~m}^{2} / \mathrm{V} \mathrm{s}$ (see $\left.[12,13]\right)$.

\section{2) PP and effect of different filler concentration}

Figure 3 shows the effect of rising concentration of nanofiller in two different PP-based cable-grade materials under a poling field of $50 \mathrm{kV} / \mathrm{mm}$, having two different base material blends and different nanofiller con concentration. As can be seen, an increase of bulk space charge at the poling field level is observed as the nanofiller concentration increases from $1 \%$-wt to $4.5 \%$-wt. It seems that for the used nanofiller and a given functionalization process, higher concentration of filler $(4.5 \%$ wt) increases the amount of accumulated charge. In fact, lower nanofiller concentration, i.e. $1 \%$-wt, shows improved space charge characteristics, reducing the amount of stored charge after three hours of polarization from $5.5 \mathrm{C} / \mathrm{m}^{3}$ to $2.5 \mathrm{C} / \mathrm{m}^{3}$ for materials from the first polymeric matrix, and from $6.2 \mathrm{C} / \mathrm{m}^{3}$ to $1.7 \mathrm{C} / \mathrm{m}^{3}$ for the ones from the second polymeric base. In the case of second matrix composition filled with $1 \%$-wt of filler, no positive slow charge packets crossing the insulation is observed. This may contribute to the reduction of the maximum field enhancement measured with such material. Similarly to the previous discussion regarding capacitor-grade materials, such 
results suggest a modification of the trap depths. Calculations on apparent mobility derived from the depolarization trends show that shallower traps are found for materials loaded with a $1 \%$-wt filler. Again, this is an important characteristic for DC cable materials, since lower trap depth distribution contributes to a quicker stored charge removal and restoration of the material natural charge neutrality (which is beneficial, in particular, in case of voltage polarity inversion). Another important result arises from the comparison with the reference XLPE performance. PP-based materials with the composition $\mathrm{B}$ feature a sensible improvement regarding space charge accumulation, when the proper filler percentage is introduced.

\section{3) Effect of different filler functionalization}

Figure 4 shows the effect on space charge patterns of the two different nanofiller functionalization processes described above. The base polymers investigated are the same introduced above, with a filling percentage of $1 \%$-wt. It can be noticed that a reduction of space charge accumulation is found in specimen whose filler went through functionalization with polar compounds (method 1). The second treatment (method 2) bring to the presence of both positive and negative charge packets crossing the insulation and eventually settling at the insulation/electrode interface, causing local field enhancement. Indeed, at the end of the polarization period, local fields of up to $79 \mathrm{kV} / \mathrm{mm}$ are experienced by materials when no functional groups are induced onto the surface of the filler, while functionalizing it with the first method up to $61 \mathrm{kV} / \mathrm{mm}$ are experienced, and $65 \mathrm{kV} / \mathrm{mm}$ with the second method. The better performance of polar functionalization comes out also by higher apparent mobilities in depolarization. Trapped charge is released, in fact, more rapidly with functionalization method $\mathrm{A}$, and the percentage of residual charge after $1800 \mathrm{~s}$ of depolarization is generally lower than for $\mathrm{B}$.

\section{CONCLUSIONS}

In spite of the project being just at the beginning, interesting results have been obtained already, indicating that thermoplastic insulation technology can be beneficial for DC apparatus in energy assets. There is a clear indication that the use of nanofiller can lead to cable designs featuring higher electric fields and temperatures, compared to the present solution. There are, however, also potential critical issues related to techniques for nanofiller functionalization and weigth percentage.
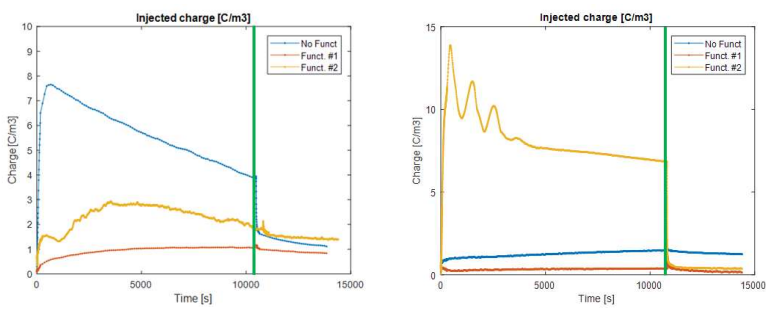

Fig. 4. Charge accumulation for two polymeric matrix compositions (A and B) with the same content of filler (1\%-wt) and different surface functionalization methods, under $50 \mathrm{kV} / \mathrm{mm}$ poling field at $60^{\circ} \mathrm{C}$. Depolarization periods starts in correspondence with the green line

\section{ACKNOWLEDGMENT}

GRIDABLE is a consortium consisting of 3 industrial partners (GE Grid Solutions, Nexans and Terichem Tervakoski), 1 consulting company (InnoEXC), 1 research institute (VTT) and 3 universities (University of Bologna, University of Tampere and University of Twente). This project has received funding from the European Union's Horizon 2020 research and innovation programme under grant agreement No 720858 .

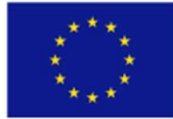

\section{REFERENCES}

[1] D. Fabiani, G. C. Montanari and L. A. Dissado, "Space charge accumulation due to ultra-fast charge packets in XLPE insulated cables: the effect of temperature and field", IEEE Inter. Conf. Properties Applications Dielectr. Materials (ICPADM), pp. 337-340, 2009.

[2] S. Delpino, D. Fabiani, G. C. Montanari, L. A. Dissado, C. Laurent and G. Teyssedre, "Fast charge packet dynamics in XLPE insulated cable models", IEEE Conf. Electr. Insul. Dielectr. Phenomena (CEIDP), pp. $421-424,2007$

[3] Tanaka, T; Bulinski, A; Castellon, J; at al., "Dielectric properties of XLPE/SiO2 nanocomposites based on CIGRE WG D1.24 cooperative test results“, IEEE Transactions on Dielectrics and Electrical Insulation, 2011, Volume 18, Issue 5

[4] Wang, Xia; Lv, Zepeng; Wu, Kai; et al., "Study of the factors that suppress space charge accumulation in LDPE nanocomposites", IEEE Transactions on Dielectrics and Electrical Insulation, 2014, Volume 21, Issue 4

[5] Lau, K. Y; Vaughan, A. S; Chen, G; et al., "Space charge dynamics in silica-based polyethylene nanocomposites", 2013 IEEE International Conference on Solid Dielectrics (ICSD), 2013

[6] G. C. Montanari, "The electrical degradation threshold of polyethylene investigated by space charge and conduction current measurements", IEEE Trans. Dielectr. Electr. Insul., Vol. 7, N. 3, pp. 309-315, 2000.

[7] G.C. Montanari, "Notes on theoretical and practical aspects of polymeric insulation aging", IEEE Electrical Insulation Magazine, Vol. 29, n. 4, pp. 30-40, August 2013.

[8] A. Cavallini, D. Fabiani, G. Mazzanti, G.C. Montanari, "Life model based on space-charge quantities for HVDC polymeric cables subjected to voltage-polarity inversions", IEEE Trans. on Dielectrics and Electrical Insulation, Vol. 9, n. 4, pp. 514-523, August 2002.

[9] G.C. Montanari, "Bringing an insulation to failure: the role of space charge", IEEE Trans. on Dielectrics and Electrical Insulation, Vol. 18, n. 2, pp. 339-364, April 2011.

[10] G. C. Montanari and G. Pattini, "Thermal Endurance Evaluation of Insulating Materials: A Theoretical and Experimental Analysis," in IEEE Transactions on Electrical Insulation, vol. EI-21, no. 1, pp. 69-77, Feb. 1986.

[11] Rytöluoto, K. Lahti, M. Karttunen, M. Koponen, S. Virtanen, and M. Pettersson, "Large-area dielectric breakdown performance of polymer films - Part II: Interdependence of filler content, processing and breakdown performance in polypropylene-silica nanocomposites," IEEE Trans. Dielectr. Electr. Insul., vol. 22, no. 4, pp. 2196-2206, Aug. 2015.

[12] K. Y. Lau, A. S. Vaughan, and G. Chen, "Nanodielectrics: Opportunities and Challenges," IEEE Electr. Insul. Mag., vol. 31, no. 4, pp. 45-54, 2015.

[13] Roy, M., Nelson, J. K., MacCrone, R. K., \& Schadler, L. S. (2007). Candidate mechanisms controlling the electrical characteristics of silica/XLPE nanodielectrics. Journal of Materials Science, 42(11), 37893799

[14] G. Mazzanti, G. C. Montanari, and J. M. Alison, "A space-charge based method for the estimation of apparent mobility and trap depth as markers for insulation degradation-theoretical basis and experimental validation," IEEE Trans. Dielectr. Electr. Insul., vol. 10, no. 2, pp. 187-197, Apr. 2003.

[15] G.C. Montanari, P. Seri, M. Ritamäki, K. Lahti, I. Rytöluoto, and M. Paajanen, "Performance of Nanoparticles in the Electrical Behavior of DC Capacitor Films" ICPADM 2018 transactions, 2018 